\title{
The Reproducibility of Patient Setup for Head and Neck Cancers Treated with Image-Guided and Intensity-Modulated Radiation Therapies Using Thermoplastic Immobilization Device
}

\author{
Akihiro Nakata ${ }^{1,2}$, Kunihiko Tateoka ${ }^{1,3^{*}}$, Kazunori Fujimoto ${ }^{1,4}$, Yuichi Saito ${ }^{1}$, Takuya Nakazawa ${ }^{1}$, \\ Tadanori Abe ${ }^{1}$, Masaki Yano ${ }^{1}$, Koichi Sakata ${ }^{1,3}$ \\ ${ }^{1}$ Department of Medical Physics, Graduate School of Medicine, Sapporo Medical University, Sapporo, Japan \\ ${ }^{2}$ Nikko Memorial Hospital, Muroran, Japan \\ ${ }^{3}$ Department of Radiology, Sapporo Medical University, Sapporo, Japan \\ ${ }^{4}$ Radiation Therapy Research Institute, Social Medical Corporation Teishinkai, Sapporo, Japan \\ Email: "tateoka@sapmed.ac.jp
}

Received July 30, 2013; revised August 25, 2013; accepted September 10, 2013

Copyright (C) 2013 Akihiro Nakata et al. This is an open access article distributed under the Creative Commons Attribution License, which permits unrestricted use, distribution, and reproduction in any medium, provided the original work is properly cited.

\begin{abstract}
The reproducibility of patient setup is an important issue for head and neck cancers treated with intensity-modulated radiation therapy (IMRT). In this study, an image-guided radiation therapy (IGRT) system has been used to minimize the uncertainty of patient setup while standard thermoplastic masks were used to provide adequate immobilization for the head and neck. However, they do not provide sufficient immobilization of the shoulders, which is an important requirement in comprehensive nodal irradiation. Therefore, we investigated the setup and rotational shifts in head and neck cancer patients undergoing IMRT for which this immobilization device had been used together with an IGRT system. The setup and rotational shifts of patients were analyzed using the ExacTrac X-ray 6D IGRT system. The patients were classified as having head and neck tumors in the upper or lower regions. The upper neck nodes included lymph nodal level II while the lower neck nodes included lymph nodal levels III and IV. Clinical data from 227 treatment sessions of 12 head and neck cancer patients were analyzed. The random translational error in inter- and intra-fraction errors of the anterio-posterior (AP) direction might influence the rotational errors of pitch and roll in the upper region. At the same time, the random translational error in the inter- and intra-fraction errors of the AP direction might influence the rotational error of roll in the lower region. We believe that these random translational errors should be considered during treatment. We found variability in random translational errors for different regions in the anatomy of head and neck cancer patients due to rotational shifts. Depending on the location of the primary lesion or the selected nodal treatment targets, these relative positional variations should be considered when setup and rotational shifts are corrected with IGRT systems before treatment.
\end{abstract}

Keywords: IMRT; IGRT; Radiation Therapy; Immobilization; Head and Neck Cancer

\section{Introduction}

The primary goal of radiation therapy is to deliver the desired radiation dose accurately to the desired target volume throughout the course of treatment. Technological advances in conformal radiation therapy have made it possible to tailor treatment to match the shape and position of the target, and thereby minimizing normal tissue damage to a greater extent than previously possible. One

${ }^{*}$ Corresponding author. technique, intensity-modulated radiation therapy (IMRT), has yielded significant gains in tumor control and toxicity reduction [1-3]. Another technique, image-guided radiation therapy (IGRT), has focused on the characterization and control of patient movement, organ motion, and anatomical deformation, which introduce geometric uncertainty and limit the effectiveness of high-precision treatment.

Target localization performed with appropriate technologies and frequency is a critical component of treat- 
ment quality assurance. For example, the target position relative to the beams has been inferred from surface marks on the patient's skin or through an immobilization device and verified using megavoltage radiographs of the treatment portal. With IGRT, imaging technology has made it possible to image soft tissue volumes and bones using $x$-rays $(\mathrm{kV})$ in the treatment setting. Enhancing localization practices reduces treatment errors, permits the monitoring of anatomical changes, and minimizes uncertainties that could influence clinical outcomes [4].

Analysis of the reproducibility of patient setup is an important issue for radiation treatments employing IMRT and IGRT. In particular, the standard thermoplastic mask provides adequate immobilization of the head and neck. However, it fails to provide sufficient immobilization of the shoulders which is an important requirement when IMRT and IGRT systems are used for comprehensive nodal irradiation $[5,6]$.

In Engelsman et al. [7] investigation of the intra-fraction patient motion before IMRT treatment of the head and neck, it was found that translational motion of less than $1 \mathrm{~mm}$ is possible, depending on the characteristics of the immobilization device used. However, they reported a limitation in determining the accuracy of patient setup, since their method only implemented two-dimensional (2D)/2D matching using 2D portal images and 2D digitally reconstructed radiographs (DRRs) [5,6,8]. There is an important flaw in this technique because it is not always possible to distinguish mismatches due to translational shifts from these images due to rotational displacements. Inter-fraction motion before IMRT treatment of the head and neck analyzed through 2D/2D matching of portal images and DRRs is also discussed in other literatures $[9,10]$.

Zhang et al. [6] investigated inter-fraction patient motion as the deviations between the daily computed tomography (CT) and the planning CT of a region of interest with respect to a reference point, as determined by three radiopaque markers on the thermoplastic mask before IMRT treatment of the head and neck. Inter-fraction patient motions, including translations and rotations, for three individual bony landmarks in the head and neck region were separately registered in the daily CTs with respect to their positions in the reference planning $\mathrm{CT}$. The study quantified inter-fraction patient motions in a three-dimensional (3D) manner, but they did not report intra-fraction patient motions. With the recent availability of IGRT systems, it is possible to correct the patient setup error just before treatment and/or before the delivery of each IMRT field with the patient immobilized in the treatment position. Data on the intra-factional motion of patients under specific immobilization methods will provide a measure of the reproducibility of patient setup when these devices are used. Additionally, the range of inter-fractional motion of individual treatment sites will provide an important data in order to set acceptable tolerances when these devices are used. The aim of this study was to evaluate the reproducibility of patient setup for head and neck cancers treated with image-guided IMRT using thermoplastic immobilization device.

\section{Materials and Methods}

\subsection{IGRT System}

Step-and-shoot IMRT beams having five segments Interand intra-fraction patient motions were analyzed using the ExacTrac x-ray 6D IGRT systems (BrainLAB AG, Feldkirchen, Germany) with system software version 3.5. The ExacTrac x-ray 6D system consists of infrared (IR) tracking and $\mathrm{x}$-ray components. The infrared tracking component includes two IR cameras, passive IR reflecting spheres placed on the patient or the immobilization device, and a plastic mask molded to the patient's contour. The IR cameras are rigidly mounted to the ceiling, and they emit a low IR signal that is reflected and analyzed for positional information. Patient setup can then be easily achieved by moving the couch to match the marker's position with those recorded in a CT image. The software also provides rotational offsets along three primary axes. The external markers must be positioned in a relatively stable location to achieve accurate setup. The $\mathrm{x}$-ray component consists of two floor-mounted $\mathrm{kV}$ x-ray tubes that project in an oblique angle medially, anteriorly, and inferiorly onto two corresponding amorphous silicon flat panel detectors mounted on the ceiling (Figure 1). Two stereoscopic images produced by the two $\mathrm{kV}$ x-ray tubes are obtained after the patient is initially set up with the ExacTrac (infrared) system. These images are then compared with the corresponding isocenter of each patient's 3D CT simulation images in the form of DRRs.

The software provides several options for matching the images. One of the methods is the six degrees of freedom (6D) fusion method assume that the patient is set up with rotational offsets. Corresponding DRRs are generated at fixed angles, and position adjustment in three translational and three rotational directions (6D) is performed on the DRRs to best match the x-ray images. The software then compares these DRRs with the corresponding $x$-ray images to obtain the set of DRRs with maximal similarity to the corresponding $\mathrm{x}$-ray images. The best match is thus determined, and the three translational and three rotational position variations used to generate the set of DRRs are the 6D offsets used to fuse the images. Jian et al. demonstrated in a phantom study that the maximal random error of this system was $\pm 0.6 \mathrm{~mm}$ in each direction with $95 \%$ confidence interval while systematic error of approximately $0.4 \mathrm{~mm}$ was found in the 


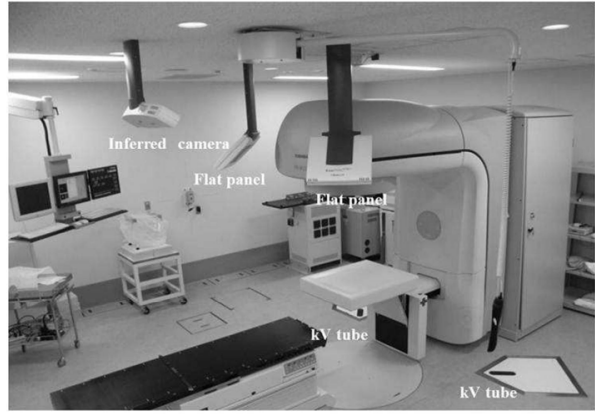

(a)

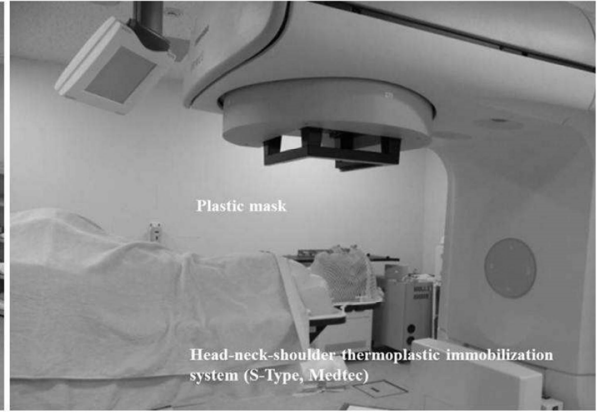

(b)

Figure 1. (a) The ExacTrac X-ray 6D IGRT systems showing the infrared camera, flat panel, and kV tube; (b) Head, neck and shoulder thermoplastic immobilization system (S-Type, Medtec, Orange City, IA) and plastic mask.

longitudinal direction [11]. Vertical, longitudinal, and lateral displacements of the table as well as couch angles (roll, pitch, and yaw) are calculated by the system to correct for the differences in the actual patient setup and the patient setup when the reference CT was taken. The entire imaging, analysis, and patient shift require 3 to 5 min of additional setup time than that needed for patients undergoing no image guidance $[8,12]$.

\subsection{Analysis of Patient Motions}

Twelve patients with head and neck cancers treated with IMRT were selected for this study. Their planning CT was taken at a spatial resolution of $0.94 \mathrm{~mm}$ per pixel in the transverse plane, and the slice thickness was $1 \mathrm{~mm}$. Before beam delivery they patient images were also taken with the ExacTrac x-ray 6D IGRT systems, of which 227 pairs of stereoscopic images were used for the study. Head and neck IMRT patients are immobilized at our institution by using a head-neck-shoulder thermoplastic immobilization system (S-Type, Medtec, Orange City, IA) with patient-specific neck cushions. IMRT treatments were performed using 6MV x-rays from a Siemens Mevatron Primus (Siemens, Munich, Germany) with 80 leaf pairs of $1 \mathrm{~cm}$ projected width at the isocenter.

For head and neck IMRT patients, we use a generic isocenter, which is typically located around the $\mathrm{C} 2$ to $\mathrm{C} 4$ vertebrae. Although individual bony structures are rigid, bony structures in the head and neck region collectively, are not simple rigid objects. Zhang et al. [6] reported that regions of interest (ROIs) in different locations in the head and neck region were needed to analyze patient motion. Patient motions were analyzed by separating the head and neck cancers into two ROIs for nasopharyngeal, oropharyngeal, and hypopharyngeal cancers. The first region was comprised of the primary tumor and upper neck nodes (upper region, close to the clivus and $\mathrm{C} 3$ vertebra (C3) regions). The upper neck nodes included lymph nodal level II of the neck. The second region was comprised of the lower neck nodes (lower region, close to the $\mathrm{C} 5$ vertebra (C5) and supraclavicular regions). The lower neck nodes included lymph nodal levels III and IV of the neck. The clivus to $\mathrm{C} 3$ region represents the tumor to subclinical disease for head and cancer patients. The C5 to supraclavicular region represents the supraclavicular lymph nodes that are occasionally treated with IMRT. Therefore, to analyze the set-up reproducibility of head and neck cancers, the region of interest was separated into upper region and lower region.

All patient setup were performed with aid of the ExacTrac system. The necessary shifts to move the patient to the isocenter were acquired with the position of the infrared markers placed on top of the thermoplastics used as bases for patient set-up localization. Stereoscopic xray images from two directions were taken. In the analysis of the upper region, the lower region of the images was masked using the system software. Likewise, in the analysis of the lower region, the upper regions in the stereoscopic images were masked as shown in Figure 2. The images were automatically registered to the DRR generated from the planning CT image set. The ExacTrac system software then computed the shifts necessary to move the patient to the calculated isocenter for upper and lower regions respectively. These were defined as the pretreatment shifts. The couch was adjusted when the computed translational and rotational shifts were more than $1.0 \mathrm{~mm}$ and 1.0 degree, respectively (the mean shift is about $1 \mathrm{~mm}$ according to rotational error of one degree in this study).

When the computed translational and rotational shifts for the upper and lower regions were in opposite directions, the patient had to be repositioned in the thermoplastic mask and stereoscopic images were re-taken to recalculate the pre-treatment shifts. Another set of stereoscopic images was taken after the completion of every fraction of treatment in order to obtain the post-treatment shifts.Inter-fractional motion, both in translational and rotational directions, was determined from all the pretreatment shifts. On the other hand, intra-fractional mo- 


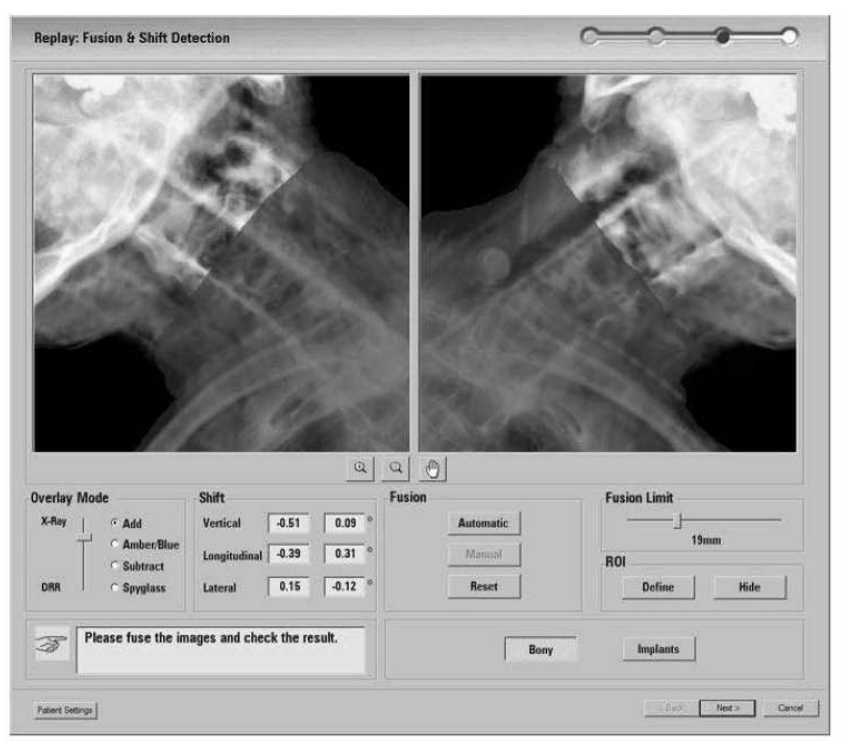

(a)

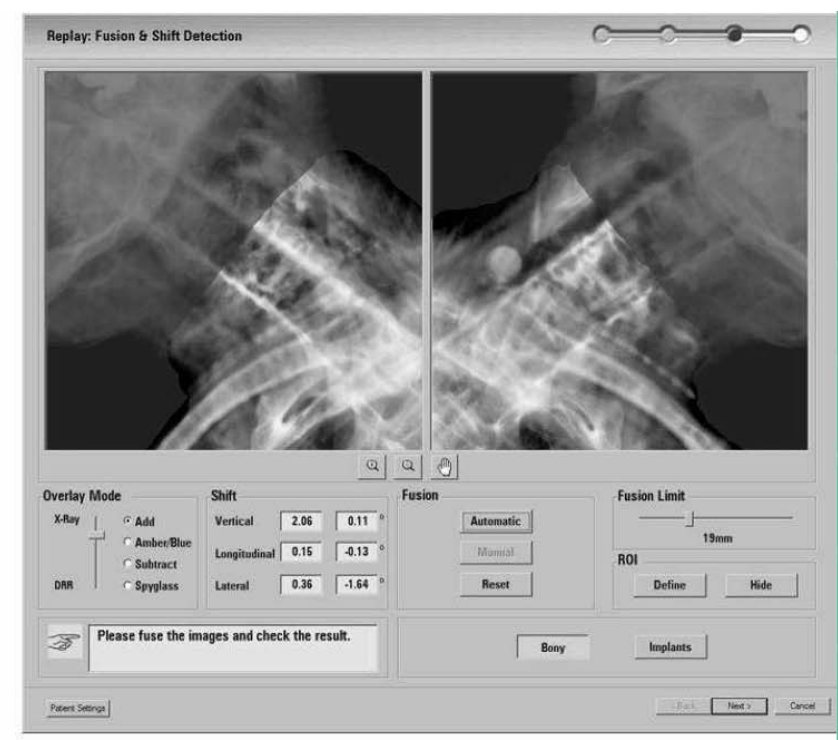

(b)

Figure 2. Two verification $\mathrm{kV}$ x-ray images for evaluating the localization accuracy, which is determined by measuring the distance of isocenter between the $x$-ray system and the treatment for the upper region (a) and lower region (b). The dark gray region not fused image is masked.

tion, both in the translational and rotational directions, was derived from the pre- and post-treatment shifts obtained within the same day (or treatment fraction). The translational shifts were calculated along the anterioposterior (AP), cranio-caudal (CC) and left-light (LR) directions while the rotational shifts in roll, pitch and yaw directions. The mean error $(\bar{X})$, systematic error $\left(\sum\right)$, and random error $(\delta)$ for the inter- and intra-fractional motions were defined as the mean of the variation inter- or intra-fractional motion of the entire patients, the standard deviation of inter- or intra-fractional motion of patient-to-patient, and the mean of the observed random standard deviations of the entire patients in the study, respectively [13].

\section{Results}

\subsection{The Mean Translational and Rotational Errors ( $\left.X_{\text {trans }}^{\text {upper }}, X_{\text {rot }}^{\text {upper }}\right)$, Systematic Errors ( $\sum_{\text {trans }}^{\text {upper }}, \sum_{\text {rot }}^{\text {upper }}$ ), and Random Errors ( $\delta_{\text {trans }}^{\text {upans }}, \delta_{\text {rot }}^{\text {upper }}$ ) for the Upper Region}

The translational errors are given in Table 1, which quantitatively summarizes the pre-treatment (Before), posttreatment (After), and intra-translation (Intra) mean $X_{\text {trans }}^{\text {upper }}$, systematic $\sum_{\text {trans }}^{\text {upper }}$, and random errors $\delta_{\text {trans }}^{\text {upper }}$ ( $\mathrm{mm}$ ) and their ranges in the LR, CC, and AP directions for the upper region of the patient population.

Table 1 shows that the translational mean and systematic error of the upper region before treatment of the entire patient population (12 patients) were small (less than $0.26 \mathrm{~mm}$ ) in all directions, indicating that there was no significant systematic error between the treatment simulation and the actual treatment. In the LR and CC directions, the translational systematic errors after treatment and the intra-fraction error were consistent (less than $0.59 \mathrm{~mm}$ ) with the pre-treatment values; however, that of the AP direction was slightly larger, with a range of 1.02 to $1.22 \mathrm{~mm}$. Moreover, the pre-treatment, post-treatment, and intra-fraction random translational errors exhibited ranges of 0.65 to $0.76 \mathrm{~mm}$ in the $\mathrm{CC}$ direction and 0.83 to $2.04 \mathrm{~mm}$ in the AP and LR directions.

The rotational errors are given in Table 2, which quantitatively summarizes the pre-treatment (Before), posttreatment (After), and intra-fraction (Intra) $X_{\text {rot }}^{\text {upper }}$, systematic $\Sigma_{\text {rot }}^{\text {upper }}$, and random errors $\delta_{\text {rot }}^{\text {upper }}$ (degree) and their ranges in the roll, pitch and yaw directions for the upper region of the patient population.

Table 2 shows that the range of the pre-treatment, post-treatment, and intra-fractionation mean rotational, rotational systematic and random rotational errors was -0.69 to 1.04 degree in the yaw and pitch directions; therefore, the effect of the roll and yaw directions was small. The range of the mean rotational error was consistent in all three directions, but the ranges of the rotational systematic and random rotational errors were larger in the pitch direction than in the roll and yaw directions. That is, the rotational errors for the upper regions were small in all directions excluding the post-treatment and inter-fraction rotational systematic and random errors in the roll direction. The systematic and random errors of translation and rotation for the upper region in all directions exhibited approximately normal probability distributions. 
Table 1. Translation error (mm) of the upper and lower regions $\bar{X}, \Sigma$ and $\delta$ are mean, systematic and random errors, respectively. (a) Before treatment; (b) After treatment; (c) Intra-translation error.

\begin{tabular}{|c|c|c|c|c|c|c|c|c|c|}
\hline & \multicolumn{3}{|c|}{ LR } & \multicolumn{3}{|c|}{$\mathrm{CC}$} & \multicolumn{3}{|c|}{$\mathrm{AP}$} \\
\hline & (a) Before & (b) After & Intra & (a) Before & (b) After & Intra & (a) Before & (b) After & Intra \\
\hline \multicolumn{10}{|c|}{ upper trans } \\
\hline$X_{\text {trans }}^{\text {upper }}$ & 0.07 & -0.07 & -0.11 & 0.07 & -0.14 & -0.18 & 0.08 & 1.02 & 1.08 \\
\hline$\sum_{\text {trans }}^{\text {upper }}$ & 0.17 & 0.36 & 0.39 & 0.10 & 0.59 & 0.58 & 0.26 & 1.07 & 1.22 \\
\hline$\delta_{\text {trans }}^{\text {upper }}$ & 0.83 & 1.17 & 1.13 & 0.65 & 0.76 & 0.65 & 1.10 & 1.79 & 2.04 \\
\hline \multicolumn{10}{|c|}{ lower trans } \\
\hline$X_{\text {trans }}^{\text {lower }}$ & 0.04 & 0.00 & 0.39 & 0.40 & 0.48 & 0.26 & 0.66 & 0.54 & 0.61 \\
\hline$\sum_{\text {trans }}^{\text {lower }}$ & 0.28 & 0.56 & 0.54 & 0.59 & 0.67 & 0.31 & 0.90 & 0.88 & 0.80 \\
\hline$\delta_{\text {trans }}^{\text {lower }}$ & 1.32 & 1.54 & 1.51 & 0.89 & 0.95 & 0.86 & 1.78 & 2.68 & 2.82 \\
\hline
\end{tabular}

Table 2. Rotational error (degree) of the upper and lower regions $\bar{X}, \Sigma$ and $\delta$ are mean, systematic and random errors, respectively. (a) Before treatment; (b) After treatment; (c) Intra-rotational error (degree).

\begin{tabular}{|c|c|c|c|c|c|c|c|c|c|}
\hline & \multicolumn{3}{|c|}{ roll } & \multicolumn{3}{|c|}{ pitch } & \multicolumn{3}{|c|}{ yaw } \\
\hline & (a) Before & (b) After & Intra & (a) Before & (b) After & Intra & (a) Before & (b) After & Intra \\
\hline \multicolumn{10}{|c|}{ upper rot } \\
\hline$X_{\text {rot }}^{\text {upper }}$ & -0.01 & -0.27 & -0.21 & 0.10 & -0.69 & -0.72 & -0.69 & 0.06 & 0.06 \\
\hline$\sum_{r o t}^{\text {upper }}$ & 0.22 & 0.46 & 0.42 & 0.18 & 1.55 & 1.54 & 0.09 & 0.14 & 0.11 \\
\hline$\delta_{r o t}^{u p p e r}$ & 0.97 & 0.84 & 1.04 & 0.81 & 1.07 & 1.03 & 0.61 & 0.75 & 0.82 \\
\hline \multicolumn{10}{|c|}{ lower rot } \\
\hline$X_{r o t}^{\text {lower }}$ & 0.32 & 1.12 & 0.28 & 1.10 & 1.12 & 0.34 & 0.25 & 0.14 & 0.16 \\
\hline$\sum_{r o t}^{\text {lower }}$ & 0.44 & 0.49 & 0.37 & 1.60 & 1.63 & 0.48 & 0.29 & 0.20 & 0.23 \\
\hline$\delta_{r o t}^{\text {lower }}$ & 1.07 & 1.15 & 1.13 & 1.59 & 1.64 & 1.53 & 0.95 & 1.00 & 0.80 \\
\hline
\end{tabular}

\subsection{The Mean Translational and Rotational Errors ( $\left.X_{\text {trans }}^{\text {lowe }}, X_{\text {rot }}^{\text {lower }}\right)$, Systematic Errors ( $\Sigma_{\text {trans }}^{\text {lower }}, \Sigma_{\text {rot }}^{\text {lower }}$ ), and Random Errors ( $\delta_{\text {trans }}^{\text {lower }}, \delta_{\text {rot }}^{\text {lower }}$ ) for the Lower Region}

The translational errors are given in Table 1, which quantitatively summarizes the pre-treatment (Before), post-treatment (After) and intra-fraction (Intra) mean $X_{\text {trans }}^{\text {lower }}$, systematic $\Sigma_{\text {trans }}^{\text {lowe }}$ and random errors $\delta_{\text {trans }}^{\text {lower }}$ $(\mathrm{mm})$ and their ranges in the LR, CC, and AP directions for the lower regions of the patient population.

Table 1 shows that the pre-treatment, post-treatment, and intra-fraction mean translational and systematic errors of the lower regions of the entire patient population (12 patients) were relatively small (less than $0.90 \mathrm{~mm}$ ) in all directions, thereby, indicating that there is no significant difference between the upper and lower regions.

In the $\mathrm{CC}$ direction, the pre-treatment, post-treatment, and intra-fraction random translational errors were similar (less than $0.95 \mathrm{~mm}$ ) to the mean translational and systematic errors; however, those of the LR and AP directions were relatively large, with a range of 1.32 to $2.82 \mathrm{~mm}$. Therefore, there were many variations between the treatment simulation and actual treatment and during treatment variables in the lower region.

The rotational errors are given in Table 2, which quantitatively summarizes the pre-treatment (Before), post-treatment (After), and intra-fraction (Intra) mean $X_{\text {rot }}^{\text {lower }}$, systematic $\Sigma_{\text {rot }}^{\text {lower }}$, and random errors $\delta_{\text {rot }}^{\text {lower }}$ (degree) and their ranges in the roll, pitch and yaw directions for the lower region of the patient population.

Table 2 shows that the range of the pre-treatment, post-treatment, and intra-fraction mean rotational and rotational systematic errors was 0.16 to 1.12 degree in the yaw and pitch directions. The ranges of the mean rotational and rotational systematic errors were similar in the yaw, pitch, and roll directions, whereas the ranges of the mean rotational and rotational systematic errors were larger in the roll direction than in the yaw and pitch directions. Moreover, the post-treatment and intra-fraction random rotational errors were relatively large in all directions; these values ranged from 0.80 to 1.59 degree. The random rotational error of the lower region was larger than that of the upper region. The systematic and random errors of translation and rotation for the lower region exhibited approximately normal probability distributions in all directions. 


\section{Discussion}

In this study, we investigated the setup reproducibility in head and neck cancer therapy for two different regions using from ExacTrac X-ray 6D IGRT data acquired while patients were immobilized in the treatment position.

Recent work by Court et al. [5] and Zhang et al. [6] indicated that bony landmarks $\mathrm{C} 2, \mathrm{C} 6$, and the palatine process of maxilla (PPM) were independently chosen as alignment targets to represent these landmarks in most head and neck cancer patients. In this way, bony structures are used as landmarks for alignment in head and neck cancer patients. For this reason, it was assumed that the position of targets or avoidance structures relative to bony landmarks is consistent between fractions for head and neck cancer patients $[5,6]$. However, it may be difficult to concurrently correct patient setup errors of upper region and lower region using these three landmarks independently.

Our landmarks for patient motions were analyzed by separating the head and neck cancers into two ROIs; the first region included the primary tumor + upper neck nodes (upper region, close to the clivus and the $\mathrm{C} 3$ vertebra (C3) region), whereas the second region included the lower neck nodes (lower region, close to the $\mathrm{C} 5$ vertebra (C5) region and the supraclavicular region). In particular, the upper region (the clivus to $\mathrm{C} 3$ region) represents the subclinical representation of the tumor for nasopharyngeal, oropharyngeal, and hypopharyngeal cancers.

Zhang et al [6] reported that the range of motion in the translational direction of the bony landmarks $\mathrm{C} 3, \mathrm{C} 6$ and PPM were generally between $-0.5 \mathrm{~cm}$ to $0.5 \mathrm{~cm}$ at a $90 \%$ confidence interval. The range of motion was slightly worse for $\mathrm{C} 6$ in the LR direction at $-0.68 \mathrm{~cm}$ to $0.62 \mathrm{~cm}$ and for the PPM in the CC direction at $-0.5 \mathrm{~cm}$ to 1.15 $\mathrm{cm}$. They reported no significant difference when using the S-board or conventional facemask. Moreover, after C2 alignment using IGRT, they reported a $90 \%$ confidence range of -5.4 to $3.3 \mathrm{~mm}$ for $\mathrm{C} 6$ in each direction and -7.5 to $9.0 \mathrm{~mm}$ for PPM in each direction. The results of Court et al. [5] were similar to those of Zhang et $a l$. Therefore, they described that some patients exhibit large uncertainties in the $\mathrm{C} 6$ or supraclavicular region that were useful for evaluating patient setup shifts. Moreover, they described that the lack of significant shoulder shifts in the first week of treatment does not necessarily indicate that few/no shoulder shifts will be observed later during treatment because one patient had no shoulder shifts larger than $5 \mathrm{~mm}$ until the 32nd fraction but had large shifts for every fraction thereafter (total treatment, 35 fractions) and other patients had large shifts from the start of treatment. The method of correcting patient setup errors for the lower region is to treat the lower region using a conventional anterior field. However, Thorstad et $a l$. [14] indicated that a cold match line between conventional and IMRT fields can be a significant cause of recurrences when implemented to treat the lower region using a conventional anterior field. For that reason, planning target volume (PTV) margins are a geometric concept defined to ensure that, in the presence of setup and other uncertainties, the prescribed dose is actually delivered to the clinical target volume (CTV) is not difficult to define using the published formulations for calculating the necessary PTV basis of normal probability distribution for patient setup error [15-17]. Therefore, a correction method for head and neck cancer patients described online re-planning to correct all shifts and rotations in different regions or physically repositioning the patient in the mask $[5,6]$. These reports are critical when treatment is given using IGRT systems to be able to reposition the patient.

In our IGRT assisted evaluation of the mean translational and systematic errors, the range of motion of the upper and lower regions of head and neck cancer patients were about $1 \mathrm{~mm}$ which was consistent with the other published results $[5,6]$. Our patient setup error data exhibited an approximately normal probability distribution.

The random translational error was larger than the mean translational and systematic errors for the upper and lower regions of head and neck cancer patients in all directions. This is because the translational error in the AP direction strongly influences the pitch and rolls rotations. The pitch and roll rotations are more associated with the translational error in the CC and LR directions, respectively, and yaw rotation is determined by the translational error in the LR and CC directions. Although it appears that large rotational errors were observed in all directions, the effects of these three rotations indicated that the effect of random translational error was larger than those of mean and systematic translational errors (Tables 1 and 2); the mean and systematic rotational errors were slightly influenced by the mean and systematic translational errors in the all directions. Therefore, the post-treatment and intra-fraction translational errors in the AP direction influence the rotational errors of pitch and roll in the upper region. At the same time, the posttreatment and intra-fraction translational errors in the AP direction influence the rotational error of roll in the lower region. For this reason, even if head and neck cancer patients were treated with IGRT and immobilization device, they do not provide sufficient reproducibility of patient setup for head and neck region, which is an important requirement in comprehensive nodal irradiation.

We believe that these random translational errors should be considered in the course of treatment. Our use of IGRT is straightforward and only adds approximately 5 min to the delivery of each fraction. In addition, global population data might permit the creation of a class solu- 
tion to calculate PTV margins.

\section{Conclusions}

In this study, we used the ExacTrac X-ray 6D IGRT system and two regions (the upper and lower region) analysis method to quantify the intra-fractionation error for patient setup with head and neck cancer patients who underwent fractionated external beam radiotherapy using a commercially available immobilization device.

Setup and rotational shifts in the upper and lower region for the head and neck cancer patients were analyzed. We found variability in random translational errors for different regions of the anatomy in the head and neck cancer patient as a consequence of rotational shifts. Depending on the location of the primary lesion or the selected nodal treatment targets, these relative positional variations should be considered when setup and rotational shifts are corrected with IGRT system and immobilization device before treatment.

\section{Acknowledgements}

The author(s) expresses their appreciation to Prof. Jun Takada (Radiation Protection Laboratory, Sapporo Medical University) for his support and insightful comments regarding this study. We likewise acknowledge the contributions to this work by Dr. Kenichi Tanaka (Radiation Protection Laboratory, Sapporo Medical University), Dr. Kenichi Kamo (Mathematics and Information Sciences, Sapporo Medical University).

\section{REFERENCES}

[1] M. J. Zelefskya, Z. Fuksa, L. Happersettb, H. J. Lee, C. C. Ling, C. M. Buman, M. Hunt, T. Wolfe, E. S. Venkatraman, A. Jackson, M. Skwarchuk and S. A. Leibel, "Clinical Experience with Intensity Modulated Radiation Therapy (IMRT) in Prostate Cancer," Radiotherapy and Oncology, Vol. 55, No. 3, 2000, pp. 241-249. http://dx.doi.org/10.1016/S0167-8140(99)00100-0

[2] A. Pirzkall, F. Lohr, A. Hoss, M. Wannenmacher, J. Debus and M. Carol, "Comparison of Intensity-Modulated Radiotherapy with Conventional Conformal Radiotherapy for Complex-Shaped Tumors," International Journal of Radiation Oncology, Biology, Physics, Vol. 48, No. 5, 2000, pp. 1371-1380. http://dx.doi.org/10.1016/S0360-3016(00)00772-0

[3] S. Tribius and C. Bergelt, "Intensity-Modulated Radiotherapy versus Conventional and 3D Conformal Radiotherapy in Patients with Head and Neck Cancer: Is There a Worthwhile Quality of Life Gain?" Cancer Treatment Reviews, Vol. 37, No. 7, 2011, pp. 511-519. http://dx.doi.org/10.1016/j.ctrv.2011.01.004

[4] B. Tomas, S. Rupert, N. Wilfried and D. E. Wazer, "Image-Guided IMRT," Springer Verlag-Heidelberg, Berlin, 2006.
[5] L. E. Court, L. Wolfsberger, A. M. Allen, S. James and R. B. Tishler, "Clinical Experience of the Importance of Daily Portal Imaging for Head and Neck IMRT Treatments," Journal of Applied Clinical Medical Physics, Vol. 9, No. 3, 2008, p. 2756. http://dx.doi.org/10.1120/jacmp.v9i3.2756

[6] L. Zhang, A. S. Garden, J. Lo, K. K. Ang, A. Ahamad, W. H. Morrison, D. Rosenthal, M. S. Chambers, X. R. Zhu, R. Mohan and L. Dong, "Multiple Region-of-Interest Analysis of Setup Uncertainties in Head-and-Neck Radiotherapy," International Journal of Radiation Oncology, Biology, Physics, Vol. 64, No. 5, 2006, pp. 1559-1569. http://dx.doi.org/10.1016/j.ijrobp.2005.12.023

[7] M. Engelsman, S. J. Rosenthal, S. L. Michaud, J. A. Adams, R. J. Schneider, S. G. Bradley, J. B. Flanz and H. M. Kooy, "Intra- and Interfractional Patient Motion for a Variety of Immobilization Devices," Medical Physics, Vol. 32, No. 11, 2005, pp. 3468-3474. http://dx.doi.org/10.1118/1.2089507

[8] J.-Y. Jin, F.-F. Yin, S. E. Tenn, P. M. Medin and T. D. Solberg, "Use of the Brainlabexactrac X-Rays 6D System in Image-Guided Radiotherapy," Medical Dosimetry, Vol. 33, No. 2, 2008, pp. 124-134.

http://dx.doi.org/10.1016/j.meddos.2008.02.005

[9] A. Bel, R. Keus, R. Vijlbrief and J. V. Lebesque, "Set up Deviations in Wedged Pair Irradiation of Parotid Gland and Tonsillar Tumors Measured with an Electronic Portal Imaging Device," Radiotherapy and Oncology, Vol. 37, No. 2, 1995, pp. 153-159. http://dx.doi.org/10.1016/0167-8140(95)01627-S

[10] R. Marsh, J. Balter, V. L. Evans and A. Eisbruch, "Design and Analysis of an Immobilisation and Repositioning System for Treatment of Neck Malignancies," Medical Dosimetry, Vol. 22, No. 4, 1997, pp. 293-297. http://dx.doi.org/10.1016/S0958-3947(97)00102-7

[11] J. Y. Jin, S. Ryu, K. Faber, T. Mikkelsen, Q. Chen, S. Li and B. Movsas, "2D/3D Image Fusion for Accurate Target Localization and Evaluation of a Mask Based Stereotactic System in Fractionated Stereotactic Radiotherapy of Cranial Lesions," Medical Physics, Vol. 33, No. 12, 2006, pp. 4557-4566. http://dx.doi.org/10.1118/1.2392605

[12] S. A. Arrizabalaga, L. B. Gonzalez, J. V. R. Ferrando, J. P. Peidro, D. P. Meseguer and T. G. Hemandez, "Prostate Planning Treatment Volume Margin Calculation Based on the Exactrac X-Ray 6D Image-Guided System: Margins for Various Clinical Implementations," International Journal of Radiation Oncology, Biology, Physics, Vol. 69, No. 3, 2007, pp. 936-943.

http://dx.doi.org/10.1016/j.ijrobp.2007.06.063

[13] J. C. Stroom and B. J M. Heijmen, "Geometrical Uncertainties, Radiotherapy Planning Margins, and the ICRU62 Report," Radiotherapy and Oncology, Vol. 64, No. 1, 2002, pp. $75-83$. http://dx.doi.org/10.1016/S0167-8140(02)00140-8

[14] W. Thorstad, S. Hong, A. J. Hope, P. E. Lindsay, B. Haughey, J. O. Deasey and K. Chao, "Patterns of Failure in Patients Receiving Intensity Modulated Radiation Therapy (IMRT) for Head and Neck Cancer," International Journal of Radiation Oncology, Biology, Physics, Vol. 63, 
2005, p. S74.

http://dx.doi.org/10.1016/j.ijrobp.2005.07.127

[15] M. V. Herk, P. Remeijer, C. Rasch and J. V. Lebisque, "The Probability of Correct Target Dosage: Dose-Population Histograms for Deriving Treatment Margins in Radiotherapy," International Journal of Radiation Oncology, Biology, Physics, Vol. 47, No. 4, 2000, pp. 1121-1135. http://dx.doi.org/10.1016/S0360-3016(00)00518-6

[16] E. Astreinidou, A. Bel and C. P. J. Raaijmakers, "Adequate Margins for Random Setup Uncertainties in Head-
and-Neck IMRT," International Journal of Radiation Oncology, Biology, Physics, Vol. 61, No. 3, 2005, pp. 938-944. http://dx.doi.org/10.1016/j.ijrobp.2004.11.016

[17] J. C. Stroom, H. C. J. de Boer, H. Huizinga and A. G. Visser, "Inclusion of Geometrical Uncertainties in Radiotherapy Treatment Planning by Means of Coverage Probability," International Journal of Radiation Oncology, Biology, Physics, Vol. 43, No. 4, 1999, pp. 905-919. http://dx.doi.org/10.1016/S0360-3016(98)00468-4 